\title{
Mandril de cateter na osteossíntese costal em um cão
}

\author{
Catheter needle for osteosyntesis of the rib in a dog \\ Marina Gabriela Monteiro Carvalho Mori da Cunha' ${ }^{1}$, Kleber Gomes', João Paulo Monteiro Carvalho \\ Mori da Cunha ${ }^{2}$, Ney Luis Pippi ${ }^{3}$ \& Josaine Cristina Rappeti ${ }^{4}$
}

\begin{abstract}
RESUMO
O trauma torácico é uma afecção, comum na rotina de pequenos animais, sendo que muitos desses animais necessitam de abordagem cirúrgica emergencial. O objetivo principal da estabilização torácica é permitir a adequada ventilação o mais cedo possível. Diante disso, o objetivo deste trabalho é relatar a aplicação de um mandril de cateter de acesso venoso, material de fácil acesso e baixo custo, na osteossíntese costal de um cão na rotina hospitalar. Um canino apresentando tórax paradoxal foi atendido no Hospital Veterinário da Universidade Federal de Santa Maria (HVU-UFSM). Foi realizada a osteossíntese da sexta e sétima costelas direitas, com fio mononáilon e com mandril de cateter, respectivamente. A técnica foi efetiva, ocorrendo a estabilização da parede costal no pós-operatório imediato e, após 60 dias, a fratura já havia consolidado. A utilização do mandril de cateter para a estabilização da fratura foi efetiva quando utilizada nesse caso da rotina cirúrgica. Mostrou-se um material de fácil manuseio e com uma maleabilidade adequada para sua introdução no canal medular da costela.
\end{abstract}

Descritores: implante, terapia intensiva, parede costal, cirurgia torácica.

\begin{abstract}
Thoracic trauma is a very common injury in small animal practice and most of them in need of emergency surgery. The main objective of the thoracic stabilization is to allow adequately ventilation as soon as possible. The aim of this report was to describe the use of a catheter needle, a low cost material, for costal osteosyntesis in a dog in the hospital routine. A dog with flail chest was admitted at the Veterinary Medical Teaching Hospital of the UFSM. The dog was submitted to osteosyntesis of the $6^{\text {th }}$ and $7^{\text {th }}$ right ribs with mononylon and catheter needle, respectively. The technique surgery used was effective and the stabilization of costal wall occurred at the immediate posoperatory. The fracture was consolidated after 60 days. Catheter needle was effective for stabilization of the rib fracture in this case report. The implant is easy handly and adequately malleability for it introduction into bone marrow canal of the rib.
\end{abstract}

Keywords: implant, intensive critical care, costal wall, thoracic surgery. 


\section{INTRODUÇÃO}

O trauma torácico é uma afecção muito comum em pequenos animais, chegando a $10 \%$ das lesões traumáticas [3], sendo que em um estudo realizado em gatos com trauma ortopédico, observou-se trauma torácico associado em $89 \%$ dos casos [2]. Muitas vezes, esses pacientes necessitam de abordagem cirúrgica emergencial, devendo-se instituir terapia rapidamente [4].

As lesões torácicas podem ser causadas por acidentes automobilísticos, brigas entre animais, quedas, armas de fogo e objetos penetrantes [3], sendo que as causas mais comuns de lesões penetrantes no tórax são ferimentos por mordeduras e ferimentos por arma de fogo [6].

O tórax instável, também chamado de tórax paradoxal, é diagnosticado quando, na inspiração, o local da fratura se deprime, e, no momento da expiração, há uma expansão [1]. O afundamento torácico (flail chest) ocorre em casos de danos graves da parede torácica, com fratura de, pelo menos, duas costelas consecutivas com no mínimo de dois pontos de instabilidade em cada [3].

A reconstrução da parede torácica deverá ser rígida, para que não ocorra movimento paradoxal durante a respiração [11] e também deve ficar hermeticamente fechada, para evitar o pneumotórax [8]. O principal objetivo da estabilidade do tórax é permitir a adequada ventilação, o mais rapidamente possível, no pós-operatório [1].

O objetivo deste trabalho é relatar a aplicação de um mandril de cateter de acesso venoso na osteossíntese costal de um cão na rotina hospitalar.

\section{RELATO DE CASO}

Um cão da raça Yorkshire, macho, com três anos, pesando $3,6 \mathrm{~kg}$, foi atendido com relato de mordida por outro cão de grande porte. Ao exame físico, as mucosas estavam rosadas e o animal estava consciente, porém apresentando movimento torácico paradoxal, e duas lesões penetrantes por mordedura no hemitórax direito.

Iniciou-se fluidoterapia com ringer lactato, terapia antibiótica com cefalotina sódica ${ }^{1}$ na dose de $30 \mathrm{mg} / \mathrm{kg}$, por via intravenosa e o paciente foi colocado em tenda de oxigênio por 10 minutos. Após estabilização, o paciente foi encaminhado à sala cirúrgica, onde recebeu midazolan ${ }^{2}$ na dose de $0,4 \mathrm{mg} / \mathrm{kg}$ e cloridrato de tramadol ${ }^{3}$ na dose de $2 \mathrm{mg} / \mathrm{kg}$, ambos por via intramuscular, como medicação pré-anestésica. Foi induzido com propofol ${ }^{4}$ na dose de $2 \mathrm{mg} / \mathrm{kg}$ por via intravenosa e mantido com isoflurano ${ }^{5}$, vaporizado em oxigênio a $100 \%$.

Após antissepsia, foi realizada a incisão sobre o afundamento torácico, podendo-se observar fratura oblíqua no terço proximal diafisário da sexta costela e fratura transversa no terço medial diafisário da sétima. Após descartar a presença de lesão no parênquima pulmonar, introduziu-se, em um ângulo de $30^{\circ}$, um o mandril de cateter $20 \mathrm{G}^{6}$ no segmento distal da sétima costela (Figura 1A). Os dois segmentos foram alinhados e fixou-se o mandril no segmento proximal, estabilizando a fratura
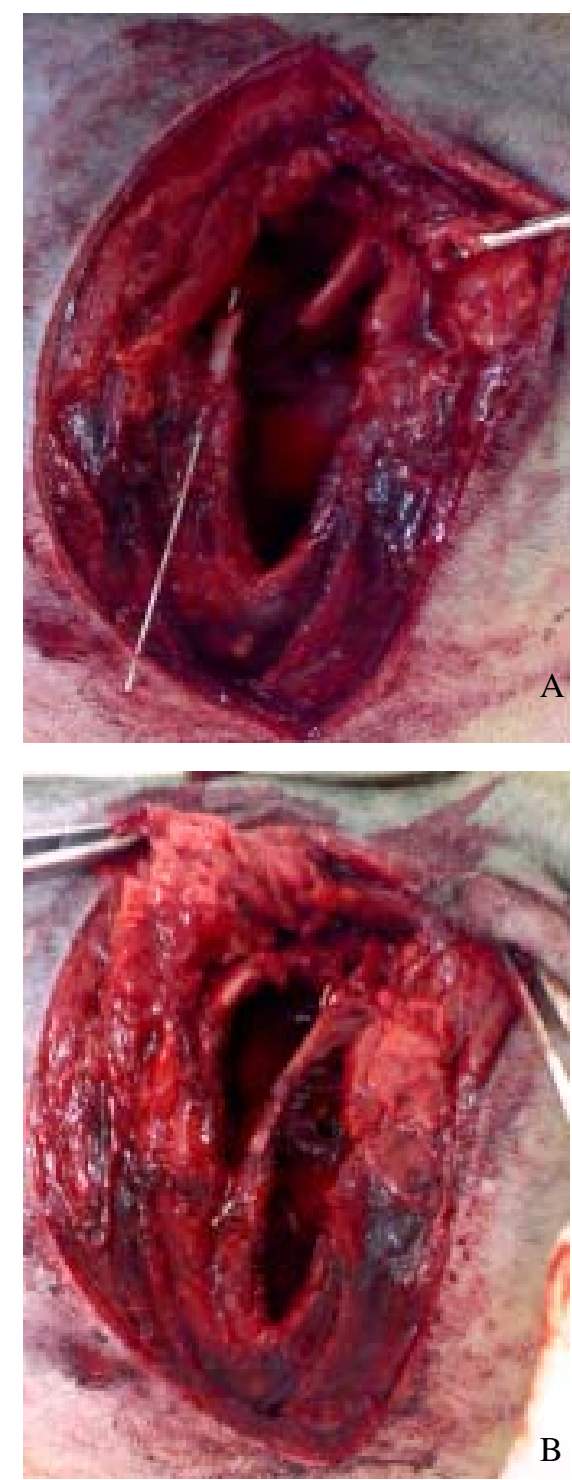

Figura 1. [A] Mandril de cateter $20 \mathrm{G}$ sendo introduzido em um ângulo de $30^{\circ}$ no segmento distal da sétima costela. [B] Aspecto final, após alinhamento dos segmentos distal e estabilização da fratura. 

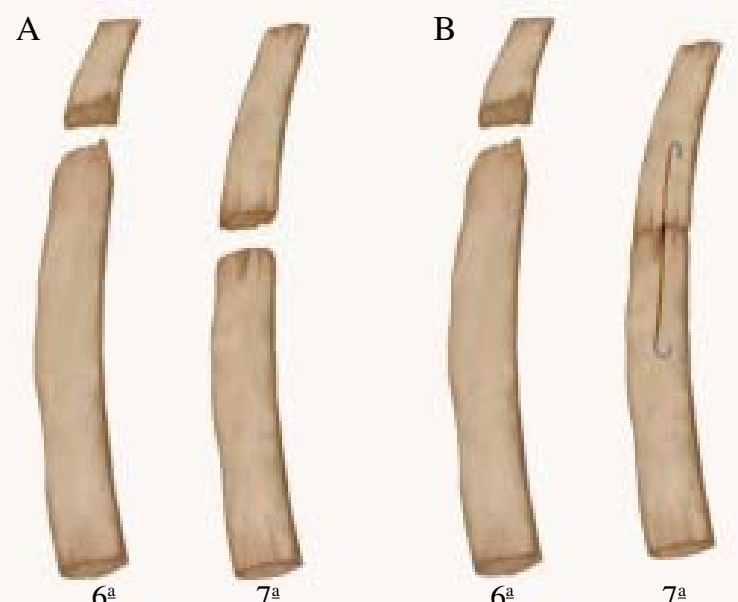

7aㅗ

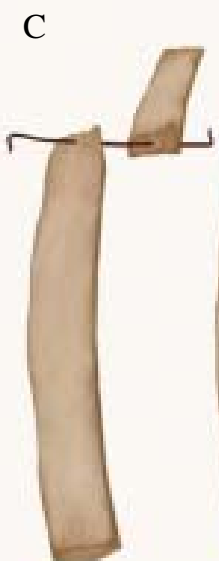

$6^{\mathrm{a}}$

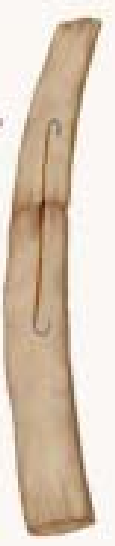

7 a

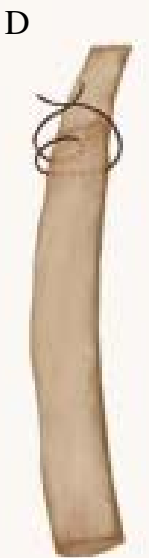

$6^{\mathrm{a}}$

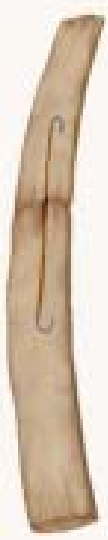

$7 \underline{a}$

Figura 2. [A] Ilustração da fratura oblíqua no terço proximal da sexta costela e da sétima costela após alinhamento dos segmentos e estabilização da fratura. [B] Confecção de um orifício em ambos fragmentos costais da sexta costela para passagem de um fio mononáilon 2-0. [C] Confecção de um ponto em oito para redução da fratura da sexta costela. (créditos Guilherme Da Cas)

(Figura 1B e 2A). As duas extremidades do mandril foram dobradas formando um gancho, para evitar migração da mesma. Na sexta costela, devido à impossibilidade da utilização de um cateter pela proximidade com a vértebra, foi realizado um orifício em ambos fragmentos para a passagem de um fio mononáilon 2-0 e confecção de um ponto em oito (Figura 2B e 2C).

A musculatura foi suturada com mononáilon 3-0 com padrão de sutura festonada. A redução do espaço morto feita com o mesmo fio em pontos isolados simples, e a pele suturada com fio mononáilon 40 . Por fim a pressão negativa foi restabelecida através da hiperinsuflação pulmonar. A restituição da pressão negativa intratorácica foi realizada por meio de toraconcentese no sétimo espaço intercostal.
No pós-cirúrgico imediato, foi realizado bloqueio intercostal com bupivacína sem vasoconstritor ${ }^{7}$ e administrado meloxican ${ }^{8}$ na dose de $0,2 \mathrm{mg} / \mathrm{kg}$ por via intravenosa e o paciente foi encaminhado para exame radiográfico em que observou-se presença do implante (Figura 3). Para analgesia pós-operatória foi utilizado tramadol, $2 \mathrm{mg} / \mathrm{kg}$ a cada $8 \mathrm{~h}$, durante 3 dias, e meloxican $0,1 \mathrm{mg} / \mathrm{kg}$ por três dias, ambos por via intravenosa. A terapia antibiótica continuou a cada 12 horas, até sete dias. $\mathrm{O}$ pino intramedular foi removido após 60 dias.

\section{DISCUSSÃO}

A avaliação radiográfica pré-cirúrgica não foi realizada, pois os animais dispnéicos descompensam muito facilmente, e não devem ser submetidos ao

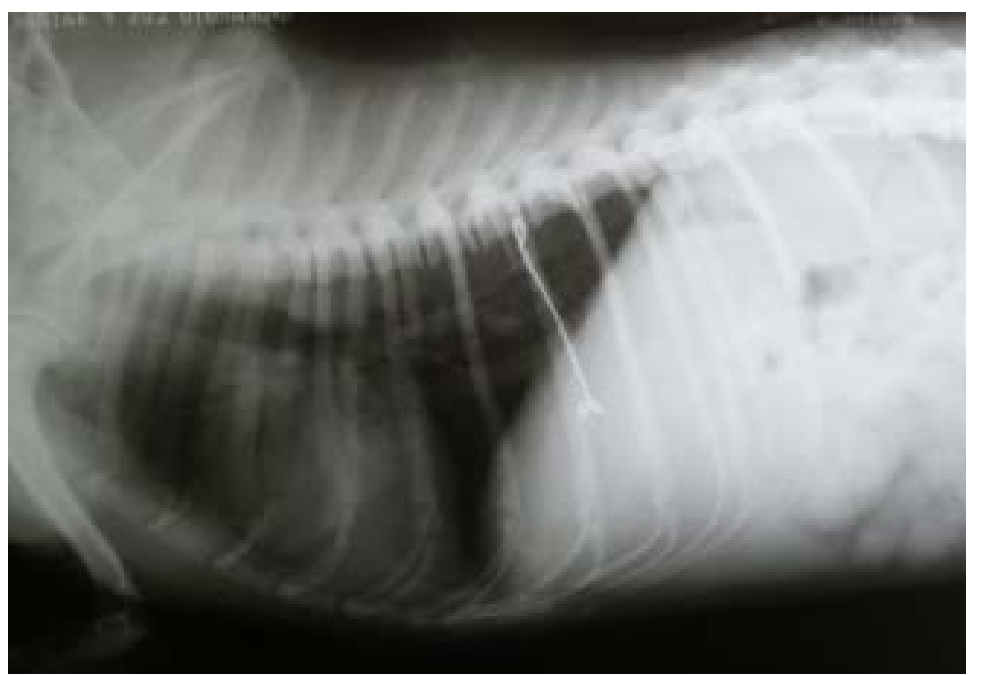

Figura 3. Exame radiográfico no pós-operatório imediato de osteosíntese da sexta e sétima costela em um cão. Notar a presença do implante no canal medular da sétima costela e a linha de fratura (seta). 
estresse antes da causa ser resolvida ou compensada [7]. A oxigenoterapia foi realizada previamente à indução anestésica, como recomendado pela bibliografia [3].

O pino utilizado para osteossíntese de costela neste relato foi o mandril de cateter, como utilizado previamente em estudo experimental em costela de cães [10]. O uso de materiais alternativos como agulhas hipodérmicas [5] e agulha de cateter [10] têm sido utilizado com sucesso em osteossínteses. Uma vantagem observada foi a sua maleabilidade que permitiu que ele se curvasse no sentido da costela. A fração da agulha inserida no segmento distal da costela foi semelhante ao tamanho no segmento proximal, sendo que a porção siliconizada do cateter serviu como guia para mensurar o tamanho introduzido no segmento ósseo distal. As extremidades do implante foram dobradas, para evitar a migração do pinos [6].

A cicatrização óssea da sexta e sétima costelas ocorreram aos 60 dias, mesmo período observado em estudo prévio [10]. Apesar de não ser recomendada a retirada do pino intramedular em costela [6], preferiu-se removê-lo, nesse caso, pois a agulha do cateter é uma estrutura que não foi fabricada com este objetivo. O gancho formado com a extremidade da agulha facilitou a identificação do implante no momento da retirada, sendo necessária a realização de duas incisões, uma dorsal e outra ventral, para a remoção de todo o mandril.
O bloqueio intercostal com bupivacína, diminui a dor no pós-operatório imediato, fornecendo uma melhor recuperação do paciente. Foi realizada a terapia antibiótica, devido à história de mordedura, em que se recomenda a utilização de antibiótico de amplo espectro [9].

\section{CONCLUSÃO}

A utilização do mandril de cateter para a estabilização da fratura, como já descrito experimentalmente por Rappeti et al., (2006) foi efetiva quando utilizada nesse caso da rotina cirúrgica. Mostrouse um material de baixo custo, de fácil manuseio e com uma maleabilidade adequada para sua introdução no canal medular da costela. Permaneceu durante todo o período pós-operatório e houve facilidade em removê-lo após a cicatrização óssea. Conclui-se, também, que o procedimento contribuiu para a sobrevida do paciente de forma significativa.

\section{NOTAS INFORMATIVAS}

${ }^{1}$ Cefalotina Sódica $500 \mathrm{mg}$ - Laboratório Teuto Brasileiro LTDA - Anápolis, GO

${ }^{2}$ Cristália Produtos Farmacêuticos LTDA - Itapira, SP

${ }^{3}$ Tramal® - Pfizer - Guarulhos, SP

${ }^{4}$ Propovan ${ }^{\circledR}$ - Cristália Produtos Farmacêuticos LTDA Itapira, SP

${ }^{5}$ Isoflurane ${ }^{\circledR}$, Cristália - Rio de Janeiro, RJ/Brasil.

${ }^{6}$ Cateter intravascular periférico Jelco, Medex, São Paulo, SP.

${ }^{7}$ Marcaína sem epinefrina - Astra Zeneca do Brasil Ltda Cotia, SP

${ }^{8}$ Maxican $0,2 \%$ solução injetável - Ourofino - Ribeirão Preto, SP.

\section{REFERÊNCIAS}

1 Aguiar E.S.V. 2001. Manual prático de emergência em pequenos animais: aspectos básicos. Porto Alegre: [s.n.]. 106p

2 Brian J.K., Kraje A.C., Rohrbach B.W., Anderson K.A., Steven B.S., Marks L.Y \& Macintire D.K. 2000. Intrathoracic and concurrent orthopedic injury associated with traumatic rib frature in cats: 75 cases (1980-1998). Journal American Veterinary Medical Association. 216: 51-54.

3 Crower JR. D.T., Shimizu R. K. \& Rabelo R.C. 2005. Trauma torácico In: Rabelo R.C. \& Crowe Jr. D.T. (Eds). Fundamentos de terapia intensiva veterinária em pequenos animais conduta no paciente crítico. Rio de Janeiro: L.F. Livros de Veterinária, p. 173-183,

4 Devey J. 2005. Paciente crítico x cirurgia emergencial como superar este desafio? Rabelo R.C. \& Crowe Jr. D.T. (Eds). Fundamentos de terapia intensiva veterinária em pequenos animais conduta no paciente crítico. Rio de Janeiro: L.F. Livros de Veterinária, p. 145-148.

5 Erdmann R.L. 2002. Utilização de materiais alternativos para cirurgias ortopédicas em aves silvestres na clínica veterinária Santa Clara, Cascavel - Paraná. In: Resumos do Congresso da Sociedade de Zoológicos do Brasil. Anais (Porto Alegre, Brasil). p. 33.

6 Fossum T.W. 2005. Endoscopia de cavidades corporais. In: Fossum T. W. (Ed). Cirurgia de pequenos animais. 2. ed. São Paulo: Roca, p. 127-132.

7 Olsen D., Renberg W., Perret J., Hauptman J.G., Waldron R. \& Monnet E. 2002. Clinical management of flail chest in dogs and cats: a retrospective study of 24 cases (1989-1999). Journal of the American Animal Hospital Association. 38: 315-320. 
8 Orton C.1998. Parede torácica. In: Slater D.S. (Ed). Manual de cirurgia de pequenos animais. 2.ed. São Paulo: Manole, p. $457-468$.

9 Plunket S.J. 2006. Emergências dermatológicas. In: Plunkett S.J. (Ed). Procedimentos de emergência em pequenos animais. 2.ed. Rio de Janeiro: Revinter, p. 80-82.

10 Rappeti J.C.S. 2006. Homoimplante de costela conservada em solução supersaturada de açúcar a 300\% ou em açúcar in natura na reconstituição experimental de costelas em gatos. 2006. 91f. Santa Maria, RS. Tese de Doutorado em Medicina Veterinária - Programa de Pós-Graduação em Medicina Veterinária, Universidade Federal de Santa Maria.

11 Ruiz A. A., Gómez H.A., Alfaro G.E. Granel C.L. \& García M.G. 1997. Sarcomas de la pared torácica. Ressección y reconstrucción. Revista Instituto Nacional de Cancerologia. 43: 189-193. 